\title{
LA CORRECTION GRAMMATICALE, LES ANOMALIES SÉMANTIQUES ET LA MÉTAPHORE DANS LA GRAMMAIRE TRANSFORMATIONNELLE-GÉNÉRATIVE
}

\begin{abstract}
A bstract. Nkollo Mikolaj, La correction grammaticale, les anomalies sémantiques et la métaphore dans la granmaire transformationnelle-générative [Grammatical Well-formedness, Semantic Anomalies and Metaphor in Transformational-Generative Grammar]. Studia Romanica Posnaniensa, Adam Mickiewicz University Press, Poznań, vol. XXVII1: 2001, pp. 83-97, ISBN 83-232-1144-2, ISSN 0137-2475.
\end{abstract}

This paper deals mainly with the problem of semantic anomalies and their role in generative grammar. Once the issue was raised, the solutions brought about by authors varied among them considerably. As far as generative grammar is a formal theory, it cannot handle the anomalous uteerances with recourse to metaphor. Instead, two main approaches were proposed. The first one originates from a semantic theory set forth by Katz and Fodor and has been adopted by Chomsky and his followers. Its focus is laid on formal properties and technical aspects of the anomalies, without trying to explore their nature and their origin. The other one, argued for by the supporters of generative semantics, has resorted to extra-linguistic context and to one's personal beliefs and utterances. Neither Chomsky's theory nor GS's theory are able to cope adequately with the problem, because its nature is strongly elusive to formal rules and its analysis can receive only several ad hoc solutions.

La linguistique générative admet que parmi les énoncés effectivement utilisés certains sont bien-formés, tandis que les autres sont agrammaticaux. La distinction est si importante qu'une des contraintes imposées à la grammaire d'une langue est d'expliquer et de prédire en quoi consiste la différence entre les uns et les autres. Vu que les locuteurs sont capables d'émettre des jugements de grammaticalité au sujet d'un ensemble infini de phrases, même inédites, c'est que cette appréciation n'a pas de caractère mnémonique ou expérimental, mais qu'elle s'appuie sur un système de règles intériorisées au cours de l'apprentissage de la langue. Donc, le linguiste, au moment où il construit sa théorie, formule des hypothèses sur les mécanismes mis en oeuvre inconsciemment par le sujet parlant. A chaque type d'agrammaticalité va alors correspondre une composante de la grammaire. En même temps, le principal enjeu de la linguistique générative consiste à permettre, au moyen d'un nombre fini d'instructions, d'engendrer toutes les phrases correctes, concevables dans une langue donnée, et elles seules. Cependant, la définition d'une phrase grammaticale n'a jamais 
été élaborée d'une faon satisfaisante tout au long de l'histoire mouvementée du développement de la théorie en question. Par conséquent, les possibilités de prévoir l'origine des combinaisons comme celles qui seront analysées dans cet article paraissent décroître au prorata de leur déviation par rapport à la norme. Du même coup, la formulation des règles censées expliquer cet état de choses s'avère une tâche ardue et semble exiger la complication de l'appareil conceptuel de la composante sémantique, ainsi que la prise en compte des mécanismes qui sous-tendent la formation des phrases (jugées) anomales.

L'observation des points de vue défendus par les représentants de la grammaire générative sur le problème d'anomalies sémantiques attire l'attention du chercheur sur deux points importants. Tout d'abord, elle permet de délimiter deux positions opposées face au problème en question, suivant que leurs auteurs accordent plus d'importance à la syntaxe ou à la sémantique. Ceci facilite, à son tour, la définition, ne fût-elle qu'approximative, d'anomalie sémantique. La première opinion, dont la paternité peut être attribuée à Katz et Fodor, consiste à déclarer qu'une phrase est anomale, lorsqu'elle est bien formée syntaxiquement et que les règles de projection sont incapables d'assigner une interprétation à l'amalgame de deux (ou plus) de ses constituants dominés par un même noeud dans un indicateur syntagmatique (cf. Katz et Postal, 1964: 26). Deux observations ont permis de rendre cette définition caduque. La première est l'existence des phrases comme C'est un contresens que de parler des pains qui mangent leurs consommateurs où la partie déviée est enchâssée dans une matrice qui «invalide» son contenu sémantique'. L'autre argument évoqué à l'encontre de cette première thèse est que, moyennant un contexte extra-linguistique approprié et un peu d'inventivité de la part des locuteurs, il est possible de conférer une interprétation sémantique aux phrases qui semblent, à première vue, «irréparables». C'est ainsi que l'énoncé comme Mon encrier a sucé ma mère dedans peut être jugé tout à fait naturelle dans un discours onirique, etc. (cf. McCawley, 1971b: 219). Les variations individuelles des sujets parlants et le bagage des expériences qu'ils mettent dans le processus d'interprétation sémantique est la carte maîtresse des partisans de cette solution. Les vices de celle-ci et la réplique de Chomsky seront discutés par la suite de cette section. Ce dernier argument, en dépit de sa stérilité due au recours aux facteurs non-linguistiques, a néanmoins servi à Chomsky (op. cit.: 152) de point de départ de la formulation de certaines lois qui permettent de hiérarchiser les écarts par rapport à la grammaticalité.

\footnotetext{
'Les possibilités d'apporter à ce type de constructions une solution appuyée principalement sur des critères transformationnels et sémantiques ont été systématiquement explorées par Chomsky (1965: 157). Selon celui-ci, la difficulté disparaît dès qu'on procède à l'inclusion des règles de sélection dans la composante syntaxique. Elle deviendra désormais apte à engendrer indirectement (derivatively) les phrases Les pains mangent leurs consommateurs, en même temps qu'elle indiquera leur statut non-grammatical. C'est maintenant aux règles de projection de prendre la relève et de «neutraliser», au moment de l'enchâssement de les pains mangent leurs consommateurs dans sa matrice parler de $+\Delta$ (le symbole postiche indique le lieu de la suite grammaticale où peut venir s'insérer la phrase constituante), les traits sémantiques opposés des constituants soumis à la combinaison.
} 
C'est ainsi que la déviation la plus grave vient du non-respect des règles de la composante catégorielle et consiste à insérer dans une suite pré-terminale le formant appartenant à la catégorie grammaticale autre que celle qu'exigent les règles syntagmatiques. C'est le cas de $l l$ a du respectueux pour la tradition. Un écart moins sensible se produit quand on contrevient aux règles de sous-catégorisation stricte qui divisent les morphèmes appartenant à une seule catégorie en sous-classes suivant les caractéristiques catégorielles de leur entourage. C'est le cas de $I l$ très respecte la tradition où l'on a affaire à l'emploi incorrect d'un adverbe d'intensité destiné à modifier les adjectifs ${ }^{2}$. Enfin, et c'est la situation à laquelle seront consacrées les réflexions qui suivent, la transgression peut résulter de la combinaison des mots aux traits sémantiques qui s'interdisent mutuellement, p. ex. La table respecte la tradition. C'est cette dernière catégorie que Chomsky qualifie de sémantiquement anomale. Ce point de vue sur la nature des expressions agrammaticales appelle qu'on y prête attention. La difficulté tient à la situation frontalière de la phrase analysée entre la syntaxe et la sémantique. La distinction [animé] vs [non-animé], censée expliquer la déviation de La table respecte la tradition se répercute, maintes fois, sur la correction (ou non) syntaxique des phrases, comme en témoignent les règles qui régissent l'emploi d'une des formes du pronom interrogatif qui / que. Si l'on se souvient de la définition que donne Chomsky de trait sémantique (,We call a feature «semantic» if it is not mentioned in any syntactic rule...", ibid.: 142), la déviation de la phrase en question a un caractère exclusivement syntaxique. Toutefois, cette opinion est certainement trop radicale. Or, sans que la définition d'anomalie proposée tout à l'heure puisse être acceptée sans méfiance, elle constitue la base sur laquelle les modifications jugées indispensables viendront se greffer.

La réalisation du postulat d'élaguer la description d'une langue des expressions qui transgressent la norme finirait par en donner une image appauvrie et éloignée de l'intuition des sujets parlants pour lesquels une multitude de phrases non grammaticales ne sont pas tout à fait incompréhensibles ${ }^{3}$. Confronté à cette difficulté, Chomsky a dû se rendre à l'évidence et il ne s'est nullement préoccupé de prouver le statut prétendument marginal des anomalies (cf. ibid.: 150, note 2; Butters, 1970: 106). En même temps il a envisagé de concéder sa place à la métaphore, censée interpréter les

\footnotetext{
${ }^{2}$ Il convient de remarquer que l'opposition entre très et beaucoup, qui explique le deuxième type de constructions non-grammaticales, se ncutralise parfois (cf. Il joue rudement bien au tennis, Il est rudement content, ll a baroquement compliqué sa description, Votre récit est baroquement compliqué, etc.), ce qui demande l'introduction dans les règles de sous-catégorisation stricte de la notion de distribution complémentaire.

${ }^{3}$ L'exemple en est la formulation des phrases référentiellement absurdes, ce qui paraît être un des procédés favoris des propagandistes. C'est ainsi qu'on a jadis parlé en Pologne de la doryphore impérialiste qui vient anéantir les plantations des pommes de terre. Sans ériger cette observation au rang d'une règle qui n'admet aucune exception, on peut dire que l'attribution à un nom d'un adjectif aux caractéristiques sémantiques opposées (à l'état libre, le terme impérialiste est destiné à se rapporter à une classe d'individus ou, secondairement, à leurs artefacts) n'étonne personne et est autorisée dès le moment où il s'agit p. ex. de produire une qualification injurieuse.
} 
combinaisons des termes qui contreviennent aux restrictions sélectives. Toutefois, selon lui, même la métaphore ne retrouve son droit de cité que dans la mesure où elle permet d'interpréter les expressions déviées grâce à leur analogie avec celles qui ont été engendrées en conformité avec toutes les règles requises de la composante de base. Cette prise de position est riche de conséquences, car, soigneux de construire une théorie à capacité générative forte, Chomsky a été amené à constater qu'elle ne doit pas se limiter à l'énumération de l'ensemble des phrases grammaticales d'une langue. Là où un écart par rapport à la norme a eu lieu, la description structurale de la phrase est tenue d'en indiquer le degré et les causes. Ces deux points - l'explication des mécanismes qui sous-tendent les expressions déviées et la légitimité du recours à la métaphore - feront l'objet de l'analyse qui suit.

En ce qui concerne l'exigence supplémentaire formulée à l'égard des descriptions structurales des phrases, qui consiste à renseigner sur les causes de la déviation et sur le degré de celle-ci, elle semble être justifiée d'une double façon. Le premier avantage qui milite en faveur d'une telle décision est lié au besoin de distinguer entre les significations des phrases anomales. Elles ne s'équivalent pas l'une à l'autre. Loin de là, certaines combinaisons dont les termes contreviennent aux restrictions sélectives sont, grâce à leur coïncidence avec une phrase bien-formée, susceptibles de recevoir une «lecture dérivée». C'est le cas de p. ex. J'ai avalé deux tomes de poésies (pouvant s'interpréter grâce à la parenté sémantique de avaler et d'une des acceptions de ingurgiter, dont les traits sémantiques et syntaxiques se recouvrent largement) qui se prête plus à l'analyse que La table respecte la tradition. Il est, et c'est le second argument qui plaide en faveur de l'indication de l'origine des phrases déviées, nécessaire de ne pas qualifier d'anomale toute expression qui s'écarte de la norme (c.à.d. ne pas croire que les anomalies sont aussi insignifiantes les unes que les autres), ceci en vue d'éviter la mise sur un pied d'égalité des sens de C'est un contresens que de parler des pains qui mangent leurs consommateurs et de C'est un contresens que de parler des tables qui respectent la tradition (cf. Jackendoff, 1972: 18). Faute de faire cette stipulation, on obtiendrait C'est un contresens que de parler des anomalies.

L'autre chose est que les règles syntagmatiques et les entrées lexicales (au sens qu'a ce terme dans la théorie de Chomsky) ne suffisent pas à prévenir la production d'une phrase incorrecte ou à rendre compte des causes qui ont prèsidé à sa formation. Même si une expression a été engendrée en conformité avec ces règles, et même si elle n'est pas, à strictement parler, anomale, elle peut susciter un certain étonnement chez les locuteurs, alors que les causes en restent méconnues. C'est ainsi que la phrase Notre entreprise possède deux grands immeubles de luxe au centre de Londres, deux gares de fret à Dover et une boîte de sardines, loin d'être incorrecte - tous les syntagmes nominaux objets, appartiennent à la même catégorie sémantique et ne different qu'au niveau de ce que Katz et Fodor (1964: 496) appellent traits différentiels - va à l'encontre de ce qui est jugé susceptible de figurer dans un échange verbal français. La seule solution capable d'en rendre compte paraît consister à introduire dans les rubriques des formants lexicaux les renseignements concernant les gabarits 
(ou la valeur pécuniaire) des objets auxquels renvoient les termes des syntagmes nominaux juxtaposés. Toutefois, ces renseignements risquent de proliférer d'une façon parasitaire et on ne voit pas le point où leur énumération pourrait s'arrễter ${ }^{4}$.

Bien que l'espoir d'aboutir à des formulations prédictives au sujet de l'origine des anomalies paraisse vain, quelques réflexions techniques qui concernent la façon dont ce type d'expressions doit être analysé s'imposent. Ces directives ont essentiellement un caractère inductif et commencent par l'observation de l'usage, donc de la performance, que font les locuteurs de leur langue. Cet usage permet d'attribuer à quelques objets, en dépit de leurs traits sémantiques inhérents, les qualificatifs qui ne leur conviennent manifestement pas, mais qui, dans des circonstances particulières, ne les rendent pas anomales. C'est le cas avec le soleil couchant dont la formation remonte à une idée précopernicienne du monde. C'est le cas encore avec la phrase de Queneau Après une attente gratinée, sous un soleil au beurre noir, je finis par monter dans un autobus pistache ou l'expression un soleil de plomb, etc. L'analyse de ces phrases suggère de ne pas voir les causes de l'anomalie là où les sujets parlants attribuent à certains objets des propriétés incompatibles avec les connaissances scientifiques (cf. aussi La distance rapetisse les objets). Elle est aussi révélatrice des rapports entre les anomalies et la vérité factuelle. Si l'expression La lune est un nombre premier est, en même temps, fausse et anomale, 16 est un nombre premier n'est clairement que fausse, sans être anomale.

L'autre perspective envisagée par Chomsky - le recours à la métaphore au moment de l'analyse des anomalies sémantiques - appelle qu'on fasse quelques remar-

\footnotetext{
${ }^{4}$ Il existe une certaine tradition en matière de l'analyse des problèmes que posent les expressions de ce type. C'est la rhétorique qui s'est depuis longtemps occupée des phrases (qu'elle appelle zeugmes) à l'intérieur desquelles les mots (ou combinaisons de mots) sémantiquement incompatibles sont mis en correspondance. C'est, p. ex., le cas avec Les clés u.ées par les siècles et par les douleurs des hommes. Bien entendu, les liens entre la rhétorique et la grammaire générative sont on ne peut plus lâches. D'autres suggestions sur la voie à adopter lors de l'explication des bizarreries sémantiques qui caractérisent les phrases comme celle dont il est question semblent remonter à Aristote. Celui-ci a postulé de ne pas opposer homme à oiseau à l'intérieur de la catégorie bipède, mais homme et bête à l'intérieur de la catégorie animal. De mềme, les boîtes de sardines et les immeubles de luxe ne s'opposent pas à l'intérieur de la catégorie des avoirs d'une entreprise. Or, peut-ềtre qu'il convient, avant de procéder à l'élaboration des classifications sémantiques, de se poser la question sur le principe qui préside à telle ou telle autre caractéristique des objets et qui est responsable des différences entre eux. Ce principe organisateur peut être la destination de certains objets (les fins qu'ils permettent de réaliser). C'est la solution, baptisée the mental questionnaire, que propose Wierzbicka (1985:333) à propos de la fameuse décomposition du champ sémantique des objets "pour s'asseoir». C'est ainsi qu'un tabouret est «sans dossier», car il permet de s'asseoir et de se pencher (p. ex. pour lacer les chaussures), alors qu'un fauteuil permet de reposer son dos (il doit donc avoir un dossier). Bien que les possibilités d'application de ce type d'analyse paraissent limitées (qu'en est-il avec les lampes, réverbères, lampadaires, veilleuses, etc. dont l'usage ne diffère pas; seuls les raisons esthétiques permettent de les opposer), elle semble résister à l'épreuve dans Notre entreprise possède deux grands immeubles de luxe au centre de Londres, deux gares de fret a Dover et une boîte de sardines. En effet, les instances où l'on emploie les immeubles, les gares de fret et les boîtes de sardines sont complètement différentes.
} 
ques pour analyser l'influence qu'elle exerce sur les principes méthodologiques de la grammaire générative. La métaphore, dont un des effets est que plusieurs sens (généralement éloignés l'un de l'autre) des termes formant une combinaison sont mis en correspondance, semble n'être qu'un expédient dans une théorie aux aspirations formelles. Dès que l'on croit possible de dériver, à partir d'un symbole initial, toutes les suites susceptibles de recevoir une interprétation sémantique et de relier ensuite celle-ci à une représentation phonétique, on doit s'interroger sur la place que doit occuper la métaphore dans la grammaire d'une langue. Or, on ne voit que peu de chances de conférer aux règles métaphoriques un même statut heuristique que celui dont jouissent les règles lexicales de la composante de base. Le rapprochement, proposé par certains auteurs (cf. Todorov, 1966: 109), entre la métaphore et la transformation d'effacement du terme censé atténuer le gradient de déviation (en l'occurence des mots et des expressions comparatifs - comme, tel, comme qui dirait, pour ainsi dire) prête flanc à deux critiques. Tout d'abord, si dans certaines situations, le parallélisme en question semble parvenir à sauver la recevabilité (Mon poisson rouge admire les toiles de Van Gogh et Mon poisson rouge fait comme s'il admirait les toiles de Van Gogh), nombreux sont les cas où l'on entrevoit ses limitations (La table fait comme si elle respectait la tradition n'explique pas grand-chose). Mais, il y a pire. Son application, au lieu de simplifier l'attribution d'une lecture à une suite anomale (mais interprétable) de formants lexicaux, finit par compliquer la tâche et produit parfois des distorsions graves (J'ai avalé deux tomes de poésies et J'ai fait conme si j'avais avalé deux tomes de poésies).

Une observation plus éclairante que celle dont il vient d'être question a été faite par Berrendonner (1995:231), cette fois-ci au sujet des verbes à argument instrumental incorporé. C'est ainsi que dans la phrase qu'il cite - Il a goudronné sa tartine d'une épaisse couche de confiture - le sentiment de bizarrerie éprouvé par le locuteur vient de la reprise, au moyen de couche épaisse de confiture, de l'information incluse dans le sens de goudronner. La conséquence en est la mise en relation de deux unités dont les sémantismes ne sont apparemment pas tout à fait compatibles entre eux. Le redoublement des arguments est, à en croire l'auteur, un des traits saillants des constructions métaphoriques qui consistent à juxtaposer deux attributs ordinairement hétérogènes. Sans aller jusqu'à nier la rectitude de ce raisonnement, il convient d'insister sur le caractère syncrétique qu'a dans cette phrase la combinaison de goudron et de couche épaisse de confiture. La distinction de leurs sens s'estompe grâce à l'effet neutralisant des traits [poisseux] et [épais], ce qui permet de conserver la cohérence sémantique de la phrase où ils figurent. Mais, la plupart du temps, une telle analyse lexicale fait défaut à la description de la nature des expressions métaphoriques. Autrement dit, la divergence entre les règles qui permettent d'engendrer «indirectement» les anomalies sémantiques et le mécanisme qui sous-tend la création de la métaphore réside dans le caractère formel des premières, mais non des secondes. Tandis que la composante de base fournit une règle (p. ex. la rubrique lexicale de ingurgiter «corrige» l'emploi de avaler dans l'exemple ci-dessus), capable de servir 
d'étalon qui représente les traits pertinents pour l'interprétation sémantique d'une phrase anomale (et permet de déceler les traits sémantiques qui ne s'accordent pas), il en est autrement avec la procédure qui donne lieu à la production des métaphores.

Celles-ci n'impliquent pas l'existence de la règle à laquelle il soit possible de recourir. En échange, elles offrent un ensemble disparate de mots unis entre eux par la contiguité (intuitivement ressentie et toujours contingente) de leurs caractéristiques sémantiques. Ceci peut être symboliquement représenté à l'aide de la séquence $\mathrm{ABCD} \vee(\mathrm{BCDE} \vee(\mathrm{CDEF} \vee(\mathrm{DEFG})))$, etc. Les lettres représentent, chacune, un trait sémantique et les combinaisons de quatre lettres symbolisent les mots de la chaîne. Dans cette perspective, la métaphore s'appuie sur la substitution, à un des sèmes du mot précédent, d'un autre sème, le reste demeurant intact. Le premier mot de la séquence est présumé représenter le sens littéral. Plus de parenthèses entourent les mots de la série, plus ils sont éloignés du sens littéral (et, par conséquent, présentent un caractère plus anomal). Il est, bien entendu, possible de faire varier le nombre de sèmes, ce qui aura pour résultat les divergences dans le type de métaphore - moins de sèmes seront pris en considération, plus éloigné de la norme en sera l'effet. Un écart plus grave doit se produire au moment de la juxtaposition des unités non-voisines (c.à.d. celles qui ont moins de sèmes en commun que les mots contigus).

Pour reprendre ce raisonnement à l'aide d'un exemple, considérons la série: beurre (substance onctueuse, comestible, peu consistante, non-dure); pâte (substance comestible, assez consistante, non-dure, peu solide); goudron (substance consistante, assez dure, assez solide, industrielle); macadam (substance dure, solide, industrielle, compressible), etc. C'est ainsi que l'emploi de ces mots avec couvrir sa tartine de est acceptable pour les deux premiers éléments de la série (objets comestibles). C'est le sens littéral. Le mot qui suit présente plus de problèmes. Ainsi, l'usage de goudron peut-il, comme on le voit dans l'exemple cité par Berrendonner, être qualifié de métaphorique. L'incorrection sémantique est ici partiellement neutralisée, ce qui résulte de la présence du trait «consistance». Celui-ci est nécessaire pour qu'un mot puisse suivre couvrir sa tartine de. En témoigne l'insertion dans le contexte en question des mots qui font penser, de même que goudron, aux substances pâteuses ou semi-liquides (colle, enduit, cf. Il a collé une couche épaisse de confiture sur sa tartine). L'aberration de couvrir sa tartine du macadam provient de l'absence dans ce mot du trait «consistance» (cf. *Il a macadamisé sa tartine d'une couche épaisse de confiture). Il en aurait été de même avec pavé, ciment, béton, etc. Ces mots, à l'opposé de goudron et de ses homologues n'ont rien à voir (sauf le trait «substance») avec les objets comestibles peu consistants dont on couvre normalement les tartines.

L'analyse qui précède permet de définir la métaphore comme l'expression à mi-chemin entre les phrases sémantiquement correctes et celles qui sont anomales. Cependant, l'emploi de cette notion en guise de concept explicatif repose sur les facteurs peu précis et peut facilement nécessiter le recours aux données non-formels et, par conséquent, inadmissibles du point de vue linguistique. Même si l'on aboutit à présenter symboliquement le mécanisme qui sous-tend la métaphore, on doit reconnaître 
que son pouvoir génératif ne garantit pas contre la formation des séries aberrantes et nettement gratuites (cf. la note 5 ci-dessous). Cet état de choses est dû à la volonté d'expliquer à chaque instant l'interprétation des unités linguistiques au moyen de deux mécanismes intellectuels indépendants: (i) mise en rapport des unités mutuellement substituables et (ii) leur adjonction aux unités lexicales coexistantes (cf. Jakobson, 1963: 61-62). Ainsi, le sens d'un mot est-il, à la fois, déterminé par l'impact de ceux qui l'entourent dans la phrase et par le souvenir de ceux qui pourraient le remplacer. Le flou qui remet en question la valeur de ce raisonnement est qu'une des relations constitutives de cette interprétation - le choix des éléments de la série - met en oeuvre tantôt les règles de sélection, tantôt la proximité sémantique (intuitivement ressentie par les sujets parlants) entre les mots de la série. Cette opération, apparemment simple et claire, se révèle à l'examen et à l'usage redoutablement imprécise, oscillant constamment entre des critères linguistiques, psychologiques ou mixtes ${ }^{5}$. Son analyse incline donc à croire que la métaphore a un caractère arbitraire et que son statut heuristique est nul. Par conséquent, elle ne peut pas jouir d'une même capacité explicative que ceci a lieu avec les règles de la structure profonde. Elle n'est pas non plus, à l'opposé du modèle génératif (susceptible de décrire plusieurs phrases potentielles), prédictive, ce qui interdit de l'incorporer au modèle.

Une fois que la métaphore est introduite dans le mécanisme formel qu'est la grammaire, il faut tenir compte de sa soumission à toutes sortes de variations individuelles, ce qui complique considérablement sa formalisation (même à supposer qu'elle soit réalisable). D'ailleurs, comme l'a remarqué Lyons (1989: 316-317), un recours excessif au sens figuré est capable de sauver n'importe quelle hypothèse, pour naive qu'elle soit. C'est la cohérence d'une théorie sémantique qui en reçoit les éclaboussures. Si ces critiques sont légitimes, elles posent les sémanticiens générativistes face à une alternative. Soit, lorsqu'il en vient au caractère de la composante sémantique, ils renoncent à son statut d'algorithme et se réclament des données intuitives et des

\footnotetext{
${ }^{5}$ Ce danger peut être illustré par le fragment de la réponse d'un schizophrène paranoïaque (sans déliquescence de ses facultés intellectuelles), demandé d'exprimer son opinion sur les causes de son séjour à l'hôpital: ,... Lekarze, którzy to zaaprobowali, to jedna klika pozostająca pod rozkazami tych z „Nafty”, naftowców, nafciarzy, nafcików, nafciuchów. To oni chcą mnie zakanistrować, zakastrować, tak jestem kastratem psychicznym, nie wierzę w żadne leki lekarzy, nie ufam ludziom, bo to jest pomacanie, pomachtanie, wermachtanie, Wehrmacht (...) Znam (...) to namawianie w knajpach, bo to jest wszystko knajpa, mówią może śledź, może kompocik, moźe bez kompociku, może herbata, może bata" (cit. d'après Masłowski et Mitarski, 1967: 107. Les italiques - M.N.). On voit que la chaîne n'embrasse pas seulement les mots apparentés phonétiquement, mais que la proximité sémantique entre les éléments de la série se laisse observer aussi (nafta-kanistrować, kompocik-herbata). Ce fragment montre non seulement les difficultés que connaissent les démarches entreprises en vue de formaliser la métaphore, mais il est aussi instructif en matière de la valeur heuristique des agrammaticalités et des anomalies sémantiques. L'étude de celles-ci est, selon Fromkin (1971:45-50), révélatrice de l'existence des règles de grammaire, en même temps qu'elle témoigne de leur réalité psychologique et illustre lesquelles de ces regles ont été transgressées. C'est, entre autres, grâce à l'observation de ce type d'énoncés qu'a été entreprise la tentative de reconstituer, en termes psycholinguistiques, la frontière formelle et normative entre ce qui est correct ct ce qui ne l'est pas.
} 
connaissances extra-linguistiques, soit ils refusent de se rabattre sur l'explication au moyen de la métaphore. En dépit des objections soulevées de part et d'autre (cf. Weinreich, 1972: 39-40. REM. Les réflexions de ce chercheur au sujet des énoncés déviés ont été formulées concurremment avec la préparation des Aspects...), c'est plutôt vers cette deuxième solution que Chomsky semble s'être tourné. La première solution, elle, paraît avoir été implicitement adoptée par les représentants de la sémantique générative au moment de leur analyse du problème des anomalies sémantiques et des contraintes sur la co-occurence.

Opposés à Chomsky qui s'inclinait à considérer les restrictions sélectives comme un type particulier de règles de la composante de base de la grammaire, ils ont présenté plusieurs arguments, parfois sains et fort pénétrants, contre cette optique pansyntaxique. Toutefois, certaines de leurs propositions doivent être soumises à un examen critique, car leur acceptation pourrait finir par battre en brèche les fondements mêmes de la grammaire générative. C'est ainsi que Lakoff (1971: 329) a constaté que la composante de base engendre les couples - phrase et présupposés - où la première n'est grammaticalement correcte que dans la mesure où elle s'accorde avec le présupposé véhiculé par le locuteur. Cette position semble être le résultat du rejet du postulat d'assigner leur interprétation sémantique à des énoncés en dehors des connaissances qu'ont les locuteurs du monde où ils vivent et elle s'appuie sur la conviction qu'il est difficile d'expliquer le sens d'une phrase sans dire ce qu'elle devient dans les différents types de situations où on peut la produire. C'est ainsi que pour celui qui considère les animaux non seulement comme des êtres vivants, mais aussi comme capables des fonctions psychologiques supérieures (p. ex. l'aptitude à avoir des sentiments), le dialogue Avec qui tu es venu? Avec mon chat, ou la phrase Ma petite pieuvre sait que je suis un piètre cuisinier sont tout à fait acceptables. Il en est de même avec d'autres animaux, tout ceci en fonction des jugements du locuteur et de la connaissance qu'il a au sujet de la réalité extra-linguistique. Ceci n'est pourtant, à s'en remettre aux arguments de Lakoff, qu'une question de performance. La compétence du locuteur, ellc. englobe l'aptitude à apparier les phrases et les présupposés en fonction desquels elles sont bien-formées. Une fois admise la définition que donne Lakoff de la correction grammaticale (et, corrélativement, d'un de ses pendants qu'est l'anomalie sémantique), on est obligé de réviser les principales thèses de la grammaire générative. Pourtant, l'erreur la plus grave que présente ce raisonnement consiste à confondre les tâches d'une théorie linguistique et les moyens qui permettent de les réaliser. La grammaire générative, de par sa nature, ne peut pas faire appel à l’intuition et espérer expliquer par là la compétence linguistique. Bien au contraire, c'est au moyen des mécanismes formels qu'elle entreprend d'expliquer les jugements intuitifs (comme celui qui concerne le statut de Ma petite pieuvre...) portés par les sujets parlants sur la nature des faits de sens. La correction (ou non) des échanges comme ceux qui viennent d'être cités n'est donc pas tributaire des jugements individuels. Ces derniers ne gardent leur validité que dans la mesure où on peut les confronter au système des règles grammaticales d'une langue donnée. Ces règles engendrent les phrases et expriment le fait qu'elles renferment certains pré- 
supposés, sans qu'il y ait besoin de faire référence à des idées particulières de qui que ce soit. Le recours aux données non-linguistiques peut, d'ailleurs, se tourner facilement contre ceux qui le pratiquent. La personnalisation des animaux aboutit à des tournures de beaucoup moins acceptables que ceci a lieu avec Avec qui tu es venu? Avec mon chat. Si les défenseurs de la thèse sur la correction relative veulent l'appliquer au pied de la lettre, ils sont obligés d'accepter, entre autres, J'achète un chat et un animal, C'est un chat, mais c'est un animal, etc.

En dépit de son caractère spécieux, l'argumentation utilisée par Lakoff a le mérite de signaler l'existence d'une catégorie d'expressions qui ont une probabilité d'occurence infime. Puisqu'elles semblent se trouver à mi-chemin entre les anomalies sémantiques et les contradictions logiques, leur statut conceptuel pose des problèmes. Les énoncés Quelques personnes sont rébarbatives, mais quelques êtres humains sont rébarbatifs ou En dépit de ma visite au Musée d'Orsay, j'y séjournais (moyennant la coréférence de Musée d'Orsay et de y) n'outrepassent nullement les lois combinatoires imposées aux phrases françaises par les traits inhérents et contextuels des unités qui y figurent. La grammaire générative, au lieu d'expliquer le statut de ces expressions à l'aide du décalage entre l'expérience et les assertions qu'elles font (c'est pourtant la solution vers laquelle tendent plusieurs chercheurs, cf. Jackendoff, op. cit.: 19-20; McCawley, 1968: 129), peut employer un concept formel et étudier les ensembles des inférences (qui interdisent de recourir à la connaissance de la réalité extra-linguistique et s'opposent, de ce fait, à C'est un castor, mais il construit des barrages ou En dépit de sa mauvaise humeur, il se met en rogne, à moins qu'on n'introduise dans les rubriques lexicales appropriées des renseignements prototypiques, p. ex. «castor - mammifère qui construit des barrages») que ces phrases permettent. Le peu d'intérêt porté par les tenants de la théorie en question aux possibilités qu'offre ce mécanisme transphrastique est le prix qu'ils doivent payer pour s'être limités uniquement à ce niveau d'analyse qu'est la phrase prise isolément ${ }^{6}$.

\footnotetext{
${ }^{6}$ Les raisons de la répugnance de Chomsky pour l'incorporation du concept de conséquence logique à la grammaire ont été exposées avant la naissance de la grammaire générative et, à ce qu'il semble, ne se sont pas désactualisées au cours du développement de cette théorie. Le principal reproche formulé par Chomsky à l'adresse des tenants de la vision logiciste des langues naturelles concerne le décalage entre celles-ci et les langues artificielles. Ce qui passe pour l'inférence dans les systèmes mathématiques ne peut être exprimé qu'en termes des listes des postulats de signification (au sens de Carnap, 1964: 423. REM. La première édition de son article a eu lieu en 1939), alors qu'aucune connaissance méthodique de la nature de cette relation n'est accessible aux chercheurs qui décrivent le discours quotidien. Si tant est qu'il soit possible de tirer, à partir de Ceci est rouge, la conséquence Ceci est coloré, c'est grâce à l'énumération préalable des inférences possibles. Il n'en est pas de même dans les langues naturelles, surtout en raison de la faible correspondance des mots de celles-ci et des particules logiques. Tandis qu'on peut légitimement, à partir de Cet homme est grand et mince, inférer Cet honme est grand, il serait bizarre de procéder d'une façon analogue avec Le drapeal polonais est blanc et rouge (cl. Chomsky, 1955: 39-41). Cette critique garde sa validité aussi en ce qui concerne la synonymie des phrases enchâssées (Tout homme qui parle français sait que l'oculiste est un médecin des yeux et Tout homme sait que l'oculiste est un médecin des yeux), argument soulevé encore plusieurs années plus tard (cf. Chomsky, 1972: 85-86 et la note 18 de son texte). Il en ressort que les langues artificielles ne sont ni des variantes plus cohérentes ni des schémas idéaux des langues naturelles.
} 
Si le recours à la logique lors de l'interprétation sémantique des phrases est constamment rejeté par Chomsky et dénoncé comme peu efficace, il ne reste au chercheur qu'à s'en remettre aux moyens qu'offre l'analyse du système lexical. Sans qu'il soit possible d'aller jusqu'à conclure au caractère général du phénomène, il convient de noter que certaines expressions jugées anomales ne le sont qu’en apparence. Comme il en a déjà été question ci-dessus, une des présomptions qui étayent cette analyse est la mise d'accent sur une certaine disparité qui s'observe au cours de l'interprétation des anomalies sémantiques; alors que les unes se laissent facilement ramener à une formulation correcte, d'autres se montrent plus récalcitrantes. Cette remarque vaut aussi pour le type dont l'esquisse va être brièvement présentée ici. Dans la phrase Le caporal hurle des ordres à ses troupes, la transgression (probablement, de moins en moins sentie par les locuteurs) vient de la difficulté que pose l'amalgame du nom doté de [+ Humain] et du cri poussé par les chiens (les loups, les chacals, etc.). La solution la plus simple consiste à chercher l'analogie entre le comportement-stéréotype d'un caporal qui, comme on a tendance à se l'imaginer, crie sauvagement après ses subordonnés et la forme acoustique que revêt le son produit par les représentants de l'espèce canine. Mais, cette interprétation paraît trop proche du savoir extra-linguistique dont l'imprécision lors de la réflexion sémantique dans la grammaire générative a déjà été dénoncée. En tout cas, elle repose sur des données intuitives et n'explique que piètrement l'origine de ce type de combinaisons. Cependant, la phrase en question peut être «corrigée» si l'on procède par le recours aux variantes stylistiques traditionnellement associées aux caporaux et aux chiens. Il existe en franais plusieurs acceptions du mot cabot, dont l'une renvoie aux chiens (style familier et péjoratif) et une autre aux caporaux (mot argotique et péjoratif, forgé à partir de caporal sous l'influence de cabot $=$ chien). Dès qu'on se rend compte de cette ressemblance, il ne reste qu'à se référer au paradigme des cris d'animaux. Il semble que ce procédé est productif, comme en témoignent, entre autres, les phrases $Q u$ 'est-ce que tu as encore à braire? (adressée à un enfant pleurnicheur), Les amoureux qui roucoulent, etc.

D'autres combinaisons sont décidément moins nombreuses et moins prévisibles, en conséquence de quoi, elles ne se prêtent pas si facilement à la correction. C'est ainsi qu'une phrase comme Jean a versé de l'espoir à Marie paraît relever du type dont l'origine se couvre d'une telle obscurité. Le contexte extra-linguistique, à moins qu'il ne soit très sophistiqué, n'y peut rien faire. Toutefois, une procédure analogue à celle qui vient d'être appliquée à propos de Le caporal hurle... s'avère être utile également dans ce cas-là, si l'on tient verser pour une variante expressive de donner. En effet, les sémantismes de chacun des verbes semblent contenir l'idée de «transmettre». C'est ainsi qu'une phrase jugée fort aberrante peut relever d'un mécanisme sémantique productif qui consiste à substituer au terme plus général son hyponyme ${ }^{7}$.

\footnotetext{
${ }^{7}$ En ce qui concerne les causes de cet état de choses, on peut observer, tout au long du système lexical d'une langue donnée, une certaine régularité qui consiste dans la plus grande liberté combinatoire d'un terme moins spécialisé par rapport à celle d'un terme dont les emplois se restreignent à un moindre
} 
Il en est de même avec Dieu insuffla la vie à sa créature qui semble s'être implanté dans la langue au moyen d'un procédé analogue. Il convient de remarquer, en même temps, que les deux phrases diffèrent l'une de l'autre quant au degré de leur acceptabilité, bien que la règle de sélection transgressée ait été la même. Il s'agit dans l'un et l'autre cas de la combinaison du verbe pourvu du trait contextuel $[-+$ Nom concret] et du nom étiqueté [non-concret]. Cependant, insuffler requiert, en guise de son complément approprié, le nom désignant un corps gazeux, senti, à s'en remettre aux jugements intuitifs des sujets parlants, comme quelque chose de moins concret qu'un liquide dont le nom semble avoir le plus de chances de se joindre à verser ${ }^{8}$.

Alors que les exemples justement discutés relèvent de la casuistique et ne prétendent nullement au statut des lois concernant les causes de la formation des phrases anomales (et de leur transfert éventuel à la catégorie de bien-formées), ils ont le mérite de constituer le point de départ de la conceptualisation des problèmes débattus ici. On vient de voir que moins un terme est spécialisé, plus il a de chances de se combiner avec celui qui se laisse décomposer en une série de traits sémantiques opposés. Cette règle s'observe avec le plus d'acuité dans les syntagmes nominaux composés de substantifs suivis de noms de couleurs. Il est possible d'expliquer au moyen de cette hypothèse l'existence en français de deux types de formations: les unes sont clairement idiomatiques et leur figement ne présente aucun doute, cf. téléphone rouge, peur bleue, matière / substance grise, (voir) des papillons noirs. Le remplacement des adjectifs ci-dessus par les noms renvoyant à une de leurs nuances aboutit à la déformation du contenu sémantique (cf. *téléphone vermillon / cramoisi / amarante, *peur violette / bleuâtre, *substance / matière grisâtre). Bien que cette thèse ait un caractère nettement spéculatif, la substitution n'aurait pas été possible, même s'il existait, dans une langue, d'autres termes neutres et renvoyant à une même étendue

domaine d'arguments. C'est ainsi que donner de l'espoir à quelqu'un et verser de l'espoir à quelqu'un renvoient à une seule situation, mais la seconde phrase n'est pas neutre stylistiquement et les rapports combinatoires que verser est susceptible de contracter jouissent de moins de liberté que ceux qui caractérisent donner, même si l'on limite l'analyse aux phrases à structure «transmettre - nom de sentiment/sensation", cf. donner l'impression d'être heureux (*verser l'impression d'être heureux), donner le vertige à quelqu'un $\left({ }^{*}\right.$ verser le vertige...), Elle lui a donné son coeur et son âme (*Elle lui a versé son coeur...). Il s'ensuit que les formules de distribution les plus fréquentes (les moins spécialisées) se fixent aux sens les plus fréquents (cf. Apresjan, 1966: 52-53).

${ }^{8}$ Selon une des hypothèses ardemment défendues par les tenants du courant dit lexique-grammaire (cf. Giry-Schneider, 1994: 12), les noms de sentiments accusent une tendance particulièrement nette à se combiner avec des verbes qui font penser aux noms de corps à l'état liquide. C'est ainsi qu'on dit être inondé de joie, nager dans l'extase, etc. Mais, il convient de signaler, en même temps, que cc phénomene n'est ni généralement répandu, ni beaucoup plus fréquent que la combinaison d'un nom avec le trait [non-concret] et d'un verbe qui exige, en guise de son sujet ou de son complément, le nom renvoyant à un gaz, cf. Cette vue anima d'un souffle ses passions, Votre déclaration a dissipé mes craintes, Tous ses espoirs sont partis / s'en sont allés en fumée, Ses suspicions se sont envolées. Il convient de noter, enfin, la présence en français de quelques cas à mi-chemin entre liquide et gazeux, cf. Son ardeur s'est évaporée, Son enthousiasme pour ce projet semble s'être volatilisé à mesure qu'il voyait l'indolence de ses collaborateurs. 
du spectre que ceux qui figurent dans les expressions en question. Les caractéristiques que présentent les locutions idiomatiques doivent être clairement opposées à celles qui s'observent dans les combinaisons d'un nom suivi de p. ex. rouge qui renvoie, selon le cas, au communisme, danger, interdiction, etc., ce qui peut, sur le plan lexical, être partiellement expliqué grâce à la notion de sèmes afférents. D'autres termes de couleurs se prêtent moins facilement à l'interprétation (cf. pink en anglais renvoie à un sympathisant non actif de la gauche $)^{9}$ et donnent lieu à plus de variations individuelles. Bien que cette deuxième variante de combinaisons en question soit moins spectaculaire que la précédente, la substitution des noms renvoyant à la nuance d'une couleur au terme général produit, la plupart du temps, une anomalie sémantique. Le mécanisme qui impose, dans la structure nom - qualificatif, le terme superordonné plutôt que son hyponyme connaît, bien entendu, des exceptions. C'est ainsi que les expressions critique incisive, scène déchirante, regard poignant, voix (son) mordant, etc. ne contiennent pas, de préférence, d'hyperonyme blesser ${ }^{10}$. Le principal problème que pose cette analyse est que, en réalité, il s'avère difficile de décider où passe la frontière entre les anomalies sémantiques et les idiotismes. Dire que les premières sont moins fréquentes et ne se rencontrent ni dans l'usage quotidien ni dans des publications lexicographiques, alors que les seconds y figurent revient à constater l'actuel état de choses, mais ne constitue pas son explication. La conclusion qui s'impose est que la plupart du temps, dans les expressions anomales à structure nom - qualificatif, plus l’adjectif est général, plus il paraît susceptible de se joindre à un nom aux caractéristiques sémantiques opposées.

Les représentants de la grammaire générative se sont toujours obstinés à prouver que les locuteurs ne se limitent pas à répéter des phrases, mais qu'ils sont capables d'en produire de nouvelles. Les règles d'interprétation sémantique et les règles lexicales ont été conues de sorte à assurer la correction des phrases au niveau de la signification, en même temps qu'il leur incombait de prédire si telle ou telle autre phrase sera anomale ou non et d'en expliquer les causes. Il y a alors un paradoxe à

\footnotetext{
${ }^{9}$ C'est de ce type d'analyse que semble relever l'interprétation de la fameuse phrase de Chomsky Des idées incolores vertes dorment furieusement (1957: 15). La juxtaposition de vert et de incolore est, bien entendu, une contradiction et n'appartient pas directement au champ d'investigation de la sémantique lexicale. Par contre, si l'idée d'être vert est associée, comme ceci se pratique parfois, à fertilité, innovation, printemps, etc., l'explication de l'expression en question présente des perspectives encourageantes. La combinaison des idées vertes dorment peut, sans qu'il faille recourir aux connaissances intuitives (et, en même temps, sans qu'on puisse y apporter des garanties scientifiques), être interprétée comme «des idées innovatrices n'ont pas encore vu le jour».

${ }^{10} \mathrm{Si}$ la tendance à construirc des énoncés potentiellement anomaux à l'aide des termes moins spécialisês plutôt qu'à l'aide de leurs hyponymes vaut aussi pour la relation verbe + complément d'objet, et ceci dans deux directions, c.à.d. dans une construction correcte: i) le verbe est moins spécialisé (cf. boire les paroles de quelqu'un, et non pas siroter / laper / déglutir les paroles de quelqu'un), ii) le complément est moins spécialisé (cf. acheter chat en poche, et non pas persan / siamois / matou); les contre-exemples ne manquent pas (dans deux directions, eux aussi): i) La police l'a épinglé, La maladie l'a cloué à son lit, Ses parents le vissent, etc. (et non pas attacher); ii) Il ne risque pas d'attraper une méningite, J'ai toujours la colique avant les examens, Vous me donnez le tournis, etc. (et non souffrance, maladie).
} 
s'apercevoir du caractère statique de ces règles, réduites à insérer les unités lexicales ayant des caractéristiques sémantiques rigides dans des suites grammaticales bien formées, ceci au moyen d'un nombre limité de traits de sélection. La conclusion qui s'impose implacablement est que le mécanisme qui sous-tend le phénomène traité un peu par-dessus l'épaule par les tenants de la théorie générative échappe à la capacité explicative de celle-ci et exhibe les insuffisances de sa composante sémantique. Avec recours à l'appareil conceptuel de la grammaire, le chercheur est voué à en rester à l'étape de l'élaboration d'une taxinomie de déviations par rapport à la norme, sans qu'il puisse arriver à en déceler les causes ou en indiquer le degré. De même, les possibilités prédictives concernant la signification offertes par la grammaire générative s'épuisent, sauf quelques cas peu nombreux, quand il en vient à décider à l'avance quel énoncé sera jugé dévié. Il ne peut pas en être autrement, car les anomalies ont ceci de particulier qu'elles ne s'établissent pas, la plupart du temps, à partir du système de règles grammaticales. Bien au contraire, elles président à la formulation des jugements au sujet de la correction sémantique d'une phrase. C'est donc par la voie intuitive qu'il est possible de décider si une règle a été bien (ou mal) appliquée et de distinguer une phrase correcte d'une anomalie, mais tout ceci rétroactivement. $\mathrm{Ce}$ dernier aspect du fonctionnement des énoncés anomaux montre pourtant l'importance qui doit être attachée à l'étude de ceux-ci, car la correction d'une expression à un état du développement de la langue ne fait souvent que consacrer les incorrections de l'époque précédente. Ceci incline à croire que les agrammaticalités sont aussi bien les signes du non-respect de certaines règles génératives que la manifestation la plus patente de l'existence de celles-ci.

\section{BIBLIOGRAPHIE}

A presjan J. (1966), Analyse distributionnelle des significations et champs sémantiques struchurés, Langages 1, pp. 44-74.

B ach E., Harms R.T. eds (1968), Universals in Linguistic Theory, Holt, Rinehart and Winston. Inc., New York.

Berrendonner A. (1995), Redoublement actanciel et nominalisations, Scolia 5, pp. 215-244.

Butters R.R. (1970), On the interpretation of ,deviant utterances", Journal of Linguistics 6 (1), Cambridge University Press, London, pp. 105-110.

Carn ap R. (1964), Foundations of Logic and Mathematics, Fodor J.A., Katz J.J., eds, pp. 419-436.

Chomsky N. (1955), Logical Syntax and Semantics: Their Linguistic Reelevance, Language 31

(1), The Linguistic Society of America, Baltimore, pp. 36-45.

Chomsky N. (1957), Syntactic Structures, Mouton, The Hague.

Chomsky N. (1965), Aspects of the Theory of Synatx, MIT Press, Cambridge, Massachusetts.

Chomsky N. (1972), Studies on Semantics in Generative Grammar, Mouton, the Hague.

Fodor J.A., Katz J.J. eds (1964), The Structure of Language. Readings in the Philosophy of Language, Prentice-Hall, Englewood Cliffs, New Jersey. 
Fromkin V.A. (1971), The Non-Anomalous Nature of Anomalous Utterances, Language 47 (1), pp. 27-52.

Giry-Schneider J. (1994), Sćlection et sémantique: problèmes et modèles, Langages 115, pp. 5-14.

Jackendoff R. (1972), Semantic Interpretation in Generative Grammar, MIT Press, Cambridge, Massachusetts.

Katz J.J., Fodor J.A. (1964), The Structure of a Semantic Theory, (in:) Fodor J.A., Katz J.J., eds, pp. 479-518.

Katz J.J., Post al P.M. (1964), An Integrated Theory of Linguistic Description, MIT Press, Cambridge, Massachusetts.

Lak off G. (1971), Presupposition and Relative Well-Formedness, (in:) Steinberg D.D., Jakobovits L.A., eds., pp. 329-340.

Ly on s J. (1989), Semantyka (trad. Wcinsberg A.), vol. 1, PWN. Warszawa.

Masłowski J., Mitarski J. (1967), Niektóre zagadnienia schizofazji, Psychiatria Polska 1, pp. 103-108.

McCawley J.D. (1968), The Role of Semantics in a Grammar, (in:) Bach E., Harms R.T., eds., pp. 86-169.

McCawley J.D. (1971), Where do noun phrases come from? (in:) Steinberg D.D., Jakobovits L.A., eds., pp. 217-231.

Steinberg D.D., Jakobovits L.A., eds (1971), Semantics. An interdisciplinary reader in philosophy, linguistics and psychology, Cambridge University Press, Cambridge.

Todorov T. (1966), Les anomalies sémantiques, Langages 1, Larousse, Paris, pp. 100-123.

Weinreich U. (1972), Explorations in Semantic Theory, Mouton, The Hague. 